\title{
On understanding of design problem formulation for compliant mechanisms through topology optimization
}

\author{
L. Cao ${ }^{1,2}$, A. Dolovich ${ }^{2}$, and W. J. Zhang ${ }^{2, *}$ \\ ${ }^{1}$ Complex and Intelligent Systems Center, East China University of Science and Technology, Shanghai, China \\ ${ }^{2}$ Mechanical Engineering, University of Saskatchewan, Saskatoon, Canada \\ *now at: Saskatoon, Canada \\ Correspondence to: W. J. Zhang (wjz485@mail.usask.ca)
}

Received: 19 March 2013 - Revised: 28 May 2013 - Accepted: 5 June 2013 - Published: 1 November 2013

\begin{abstract}
General problems associated with the design of compliant mechanisms through the topology optimization technique are defined in this paper due to the lack of comprehensive definitions for these problems in the literature. Standard design problems associated with rigid body mechanisms, i.e. function generation, path generation and motion generation, are extended to compliant mechanisms. Functional requirements and the associated 25 formulations in the literature are comprehensively reviewed along with their limitations. Based on whether the output is controlled quantitatively or not, these formulations are categorized into two types: (1) formulations for quantitative design; and (2) formulations for qualitative design. In addition, formulations that aim to solve the point flexure problem are also discussed. Future work is identified based on the discussion of each topic.
\end{abstract}

\section{Introduction}

A Compliant Mechanism (CM) is a mechanism that gains at least part of its mobility from the deformation of its flexible members (Howell, 2001). Figure 1 shows a compliant gripper, and the arrows denote the input forces which drive the flexural hinges at A, B, C to deform, thereby causing the deformation at the output port $\mathrm{D}$.

The Topology Optimization (TO) technique is an approach to determine the topology as well as shape of a mechanism or structure for desired functions using optimization techniques. The term "topology" in the context of CMs means material distribution such as holes and interconnecting segments among various points of interest including points which serve input and output forces and points on the ground object (Ananthasuresh, 1994). For instance, different numbers of holes in CMs mean different topologies. It is noted that different topologies as well as shapes have different functions according to the general knowledge framework called FCBPSS (F: function; C: context; B: behaviour; P: principle; SS: structure/state) (Lin and Zhang, 2004). In the design of CMs, different CMs, which can be understood as material distributions, fulfill the functional requirements. Thus, designers need to select the most appropriate one to fulfill the functional requirements from those distributions or design solutions in a general sense. The performances of all these solutions are analyzed to see if they meet the requirements and the one that best meets the requirements would be chosen as the final design.

In the TO, the objective function and constraints are defined to represent different design problems. Variables in the objective function and constraints represent material distributions. The Finite element model technique is applied to calculate the response of the material distributions (solutions). The solutions are further evaluated against the objective function and constraints. An optimization algorithm is employed to update the design variables to generate new topologies. In all, the basic idea of the TO is to determine the material distribution in a given design domain for specified function requirements, and optimization techniques are employed to determine the best material distribution.

Objective functions and constraints are formulated for function requirements as metrics in the TO for the design of CMs. In the literature, flexibility, stiffness, mechanical efficiency (ME), mechanical advantage (MA), geometrical 


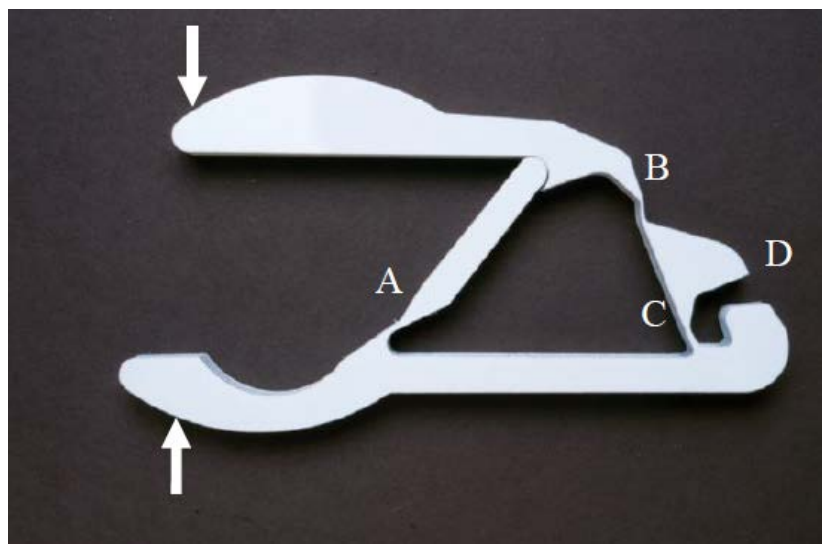

Figure 1. A compliant gripper. Courtesy of the Compliant Mechanisms Research Group at Brigham Young University.

advantage (GA), weight, strength and so on, have been defined as objective functions or constraints (Howell, 2001; Saxena and Ananthasuresh, 2001; Deepak et al., 2009). Ananthasuresh (1994) pioneered the TO of CM design with a multi-criteria model and a spring model. In both cases, a CM is desired to be sufficiently flexible to deform and sufficiently stiff to bear external loads. Formulations with different combinations of flexibility and stiffness have been studied and generalized, leading to CMs with a balance between flexibility and stiffness (Frecker et al., 1997; Saxena and Ananthasuresh, 2000). These formulations are all energy-based and originally from structural optimization which designs structures with the concepts strain energy or mutual strain energy. Moreover, the underlying logic is that we first try to find and borrow reasonable and feasible formulations from structural optimization, and then define CM design problems, accordingly. This, however, is precisely in the inverse order of the design process. As a designer, one always starts with the design problem, and then tries to search for tools or techniques (formulations in the case of the TO) to solve the problem.

Instead of formulating CM design problems with energybased concepts, Lau et al. (2001) implemented three formulations for the design of CMs based on functional requirements of mechanisms, i.e. MA, GA and ME. Luo and Zhang (2012) designed CMs with a formulation involving both ME and strain energy with input displacement constraint and dynamic response constraint. However, MA, GA and ME are all of secondary importance in terms of the functional specifications of mechanisms, and the essential functional requirements for mechanism design, e.g. displacement or force, need to be considered as the first priority.

Deepak et al. (2009) had a comparative study of five formulations, i.e. stiffness-flexibility, MA, work ratio, characteristic stiffness and artificial springs. Three design problems, namely inverters, crimpers and grippers, were implemented based on these formulations.
Wang (2009a) classified CMs into four types according to the forms of inputs and outputs: (1) displacement-displacement, (2) displacement-force, (3) force-displacement, and (4) force-force. However, design problems and formulations as defined with these attributes are not the main streams associated with mechanism design. To the contrary, the main streams for mechanism design are (1) Function Generation (FG), (2) Path Generation (PG), and (3) Motion Generation (MG). Therefore, it is necessary to critically evaluate the literature, and define design problems for CMs in such a way that they are treated as mechanism design problems from the very beginning of the process. This will provide the necessary foundation for future work in CM design.

\section{Design problems for compliant mechanisms}

A mechanism is a device which can transfer or transform force or motion (Howell, 2001). The synthesis of mechanisms is to determine the topology and geometry for desired motions (motion or force transmission) and other mechanical characteristics. Kinematics and kinetics are two aspects in respect to the synthesis. For rigid-body mechanisms, kinematics is the study of motion without involving any forces, while kinetics is the study of force-motion relations. In the design of mechanisms, desired motions are considered first, and then the forces associated with these motions are investigated using, for example, Newton's second law $(F=m a)$ to compute the force $F$ given the acceleration $(a)$ and inertia $(m)$ of the mechanism (Norton, 2003).

In rigid body kinematics, applied forces are not considered, since the motion is governed only by the geometry, material distribution, and input motions. For a $\mathrm{CM}$, however, the applied forces from actuators and/or the environment must be considered, since the motion is not only governed by the geometry and mass distribution, but also by the forces (i.e. external forces and body forces). That is, in the case of a CM, kinematics and kinetics merge. This implies that the design problem, which is generally related to the input and output ports, is always associated with both the motion and forces.

In the most general form, generalized input motions (and forces) and output motions (and forces) are described by

$X_{\mathrm{I}}^{\mathrm{T}}=\left[\boldsymbol{x}_{\mathrm{I}}^{\mathrm{T}} \boldsymbol{\theta}_{\mathrm{I}}^{\mathrm{T}}\right]$ and $X_{\mathrm{O}}^{\mathrm{T}}=\left[\boldsymbol{x}_{\mathrm{O}}^{\mathrm{T}} \boldsymbol{\theta}_{\mathrm{O}}^{\mathrm{T}}\right]$

$P_{\mathrm{I}}^{\mathrm{T}}=\left[\boldsymbol{f}_{\mathrm{I}}^{\mathrm{T}} \boldsymbol{\tau}_{\mathrm{I}}^{\mathrm{T}}\right]$ and $P_{\mathrm{O}}^{\mathrm{T}}=\left[\boldsymbol{f}_{\mathrm{O}}^{\mathrm{T}} \boldsymbol{\tau}_{O}^{\mathrm{T}}\right]$

where $X_{\mathrm{I}}, X_{\mathrm{O}}, P_{\mathrm{I}}, P_{\mathrm{O}}$ denote the inputs and outputs of a $\mathrm{CM}$, i.e. vectors of input displacements, output displacements, input driving forces and output loads (Fig. 2). Further, $\boldsymbol{x}$ and $\boldsymbol{\theta}$ denote the vectors of translational and rotational displacements while $f$ and $\tau$ denote the vectors of forces and moments (Wang, 2009a). Clearly, there could be CMs with multiple inputs and multiple outputs, but without loss 


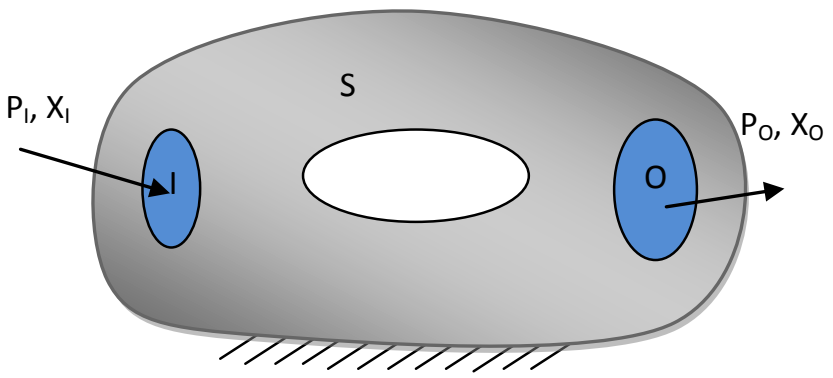

Figure 2. Inputs and outputs of a CM.

of generality, our study only focuses on CMs with a single input and a single output.

The inputs and outputs of a CM are governed by the system's static equilibrium equations in a matrix form (assuming that the inertia and damping are negligible).

$[\mathbf{K}]\left[\begin{array}{c}X_{\mathrm{I}} \\ X_{\mathrm{O}} \\ X_{\mathrm{S}}\end{array}\right]=\left[\begin{array}{c}P_{\mathrm{I}} \\ P_{\mathrm{O}} \\ P_{\mathrm{S}}\end{array}\right]$

where $[\mathbf{K}]$ is the stiffness matrix of the system and $X_{\mathrm{I}}\left(P_{\mathrm{I}}\right), X_{\mathrm{O}}$ $\left(P_{\mathrm{O}}\right), X_{\mathrm{S}}\left(P_{\mathrm{S}}\right)$ are the nodal displacements (loads) of nodes in the input port $\mathrm{I}$, the output port $\mathrm{O}$ and the remaining region $\mathrm{S}$. The stiffness matrix is not necessarily restricted to the CM, but could also contain the stiffness information of other components in the system. For example, for a system consisting of a $\mathrm{CM}$, an actuator, and a work-piece, the stiffness matrix $[\mathbf{K}]$ equals $\left[\mathbf{K}_{\mathrm{C}}\right]+\left[\mathbf{K}_{\mathrm{A}}\right]+\left[\mathbf{K}_{\mathrm{W}}\right]$ where $\left[\mathbf{K}_{\mathrm{C}}\right],\left[\mathbf{K}_{\mathrm{A}}\right],\left[\mathbf{K}_{\mathrm{W}}\right]$ are the global stiffness matrices of the $\mathrm{CM}$, the actuator, and the work-piece, respectively. Thus, Eq. (3) could represent a system of CMs, actuators and work-pieces. Note that $X_{\mathrm{S}}$ and $P_{\mathrm{S}}$ are dependent on $X_{\mathrm{I}}, P_{\mathrm{I}}, X_{\mathrm{O}}$ and $P_{\mathrm{O}}$ so that the system equilibrium equations can be re-written as:

$[\overline{\mathbf{K}}]\left[\begin{array}{l}X_{\mathrm{I}} \\ X_{\mathrm{O}}\end{array}\right]=\left[\begin{array}{c}P_{\mathrm{I}} \\ P_{\mathrm{O}}\end{array}\right]$

where $[\overline{\mathbf{K}}]$ is a transformation of the system matrix $[\mathbf{K}]$, and $X_{\mathrm{I}}, P_{\mathrm{I}}, X_{\mathrm{O}}, P_{\mathrm{O}}$ are the inputs and outputs of the system (Wang, 2009a).

The inputs and outputs of the system, i.e. $X_{\mathrm{I}}, P_{\mathrm{I}}, X_{\mathrm{O}}$ and $P_{\mathrm{O}}$, are the excitations and responses of the system governed by Eq. (4) due to the equilibrium of the system. The design problem for such a system is to determine the parameters so that the system has desired responses with specified excitations. The final designs can be different depending on the design specifications. Specifications of actuators and workpieces have significant influence on the design so that four design specifications, as shown in Table 1 , are considered to define design problems for CMs depending on whether actuators/work-pieces are considered or not in the design.

In the case (1), none of actuators and work-piece is considered in the design, which means $[\mathbf{K}]$ is just the stiffness of the $\mathrm{CM}$.
Table 1. Four Design Specifications of CM Design.

\begin{tabular}{lcc}
\hline & Without actuator & With actuators \\
\hline Without work-piece & (1) & (3) \\
With work-pieces & (2) & (4)
\end{tabular}

In the case (2), work-pieces are considered in the design while no actuator is considered, that is, $[\mathbf{K}]=\left[\mathbf{K}_{\mathrm{C}}\right]+\left[\mathbf{K}_{\mathrm{W}}\right]$. Therefore, the interaction between the output port and the loading object (or work piece) is considered. An example of this would be modeling the work-piece as a one-dimensional linear spring attached to the output port of a $C M$, so that $\left[\mathbf{K}_{\mathrm{W}}\right]$ is just the global stiffness matrix of the spring in the system.

In the case (3), actuators are considered while no workpiece is considered in the design, that is, $[\mathbf{K}]=\left[\mathbf{K}_{\mathrm{C}}\right]+\left[\mathbf{K}_{\mathrm{A}}\right]$. In the literature, for example, the actuator is simplified as a one-dimensional linear spring with an applied force.

In the case (4), both actuators and work-pieces are considered in the design, that is, $[\mathbf{K}]=\left[\mathbf{K}_{\mathrm{C}}\right]+\left[\mathbf{K}_{\mathrm{A}}\right]+\left[\mathbf{K}_{\mathrm{W}}\right]$. The interactions between the $\mathrm{CM}$ and the actuators and work-pieces are considered.

In what follows, design problems of CMs are defined based on these four cases. The inputs and outputs of a CM, i.e. $X_{\mathrm{I}}, P_{\mathrm{I}}, X_{\mathrm{O}}$ and $P_{\mathrm{O}}$, are used to describe the design problems, so they are called "problem state variables".

\subsection{Case (1)}

For the analysis of a CM in the Case (1), $P_{\mathrm{O}}$ has to be specified. In addition, there are four problem state variables and two governing equations for the system, as shown in Eq. (4), so that one more problem state variable (one of $X_{\mathrm{I}}, P_{\mathrm{I}}, X_{\mathrm{O}}$ ) needs to be specified as well to solve the system.

For the design of a CM in the Case (1), the output displacement is desired to achieve $X_{\mathrm{O}}^{*}$, i.e. $X_{\mathrm{O}}=X_{\mathrm{O}}^{*}$. In other words, in Eq. (4), either $X_{\mathrm{I}}$ or $P_{\mathrm{I}}$ could be the known problem state variable so that the system can be solved. It is noted that by considering "known" there are two situations: (a) being prescribed or given or (b) being a design variable to achieve a "best" objective. The same interpretation of "known" is also true for the case (2), (3) and (4). With the foregoing discussion, design Problems (DPs) can now be stated as follows:

- DPA: given $X_{\mathrm{I}}$ and $P_{\mathrm{O}}$, design a CM so that its $X_{\mathrm{O}}$ achieves $X_{\mathrm{O}}^{*}$.

- DPB: given $P_{\mathrm{I}}$ and $P_{\mathrm{O}}$, design a CM so that its $X_{\mathrm{O}}$ achieves $X_{\mathrm{O}}^{*}$.

Note that any one or two of the $P_{\mathrm{I}}$ and $X_{\mathrm{I}}$ could also be unknown (unknowns) and need to be optimally determined. For example, given $P_{\mathrm{O}}$, design a $\mathrm{CM}$ and optimally determine its corresponding $X_{\mathrm{I}}$ so that its $X_{\mathrm{O}}$ can achieve $X_{\mathrm{O}}^{*}$. It is obvious that, for different inputs (magnitudes or directions or locations), the results of the TO might be very different. Thus, 
considering inputs as design variables could lead to more spaces of solutions. Optimally determining inputs does not only mean the optimal determination of input magnitudes, but also directions or locations. These design problems are not listed but they can be easily extended based on the basic design problems, e.g. DPA or DPB. This treatment can also be found in other cases and will not be mentioned again unless necessary.

\subsection{Case (2)}

For the analysis of a CM in the Case (2), the output force and output displacements are coupled by the work-piece whose global stiffness $\left[\mathbf{K}_{\mathrm{W}}\right]$. Therefore, one of the problem state variables $X_{\mathrm{I}}, P_{\mathrm{I}}, X_{\mathrm{O}}, P_{\mathrm{O}}$ should be known to solve the system.

For the design of a $\mathrm{CM}$ in case the (2), either $X_{\mathrm{O}}=X_{\mathrm{O}}^{*}$ or $P_{\mathrm{O}}=P_{\mathrm{O}}^{*}$ could be the desired output. Either $X_{\mathrm{I}}$ or $P_{\mathrm{I}}$ could be the known problem state variable so that the system can be solved. Design problems are stated as follows:

- DPC: given $X_{\mathrm{I}}$ and $\left[\mathbf{K}_{\mathrm{W}}\right]$, design a CM so that its $X_{\mathrm{O}}$ achieves $X_{\mathrm{O}}^{*}$.

- DPD: given $P_{\mathrm{I}}$ and $\left[\mathrm{K}_{\mathrm{W}}\right]$, design a CM so that its $X_{\mathrm{O}}$ achieves $X_{\mathrm{O}}^{*}$.

- DPE: given $X_{\mathrm{I}}$ and $\left[\mathbf{K}_{\mathrm{W}}\right]$, design a CM so that its $P_{\mathrm{O}}$ achieves $P_{\mathrm{O}}^{*}$.

- DPF: given $P_{\mathrm{I}}$ and $\left[\mathrm{K}_{\mathrm{W}}\right]$, design a CM so that its $P_{\mathrm{O}}$ achieves $P_{\mathrm{O}}^{*}$.

Note that any one or two or three of the $P_{\mathrm{I}}, X_{\mathrm{I}}$ and $\left[\mathbf{K}_{\mathrm{W}}\right]$ could also be unknown (unknowns) and need to be optimally determined. By optimally determining $\left[\mathbf{K}_{\mathrm{W}}\right]$, the global stiffness matrix of the work-piece, we actually determine the local stiffness matrix of the work-piece and the location of the work-piece.

\subsection{Case (3)}

For the analysis of a $\mathrm{CM}$ in the case (3), $P_{\mathrm{O}}$ due to the loadingobject has to be specified and either $X_{\mathrm{I}}$ or $P_{\mathrm{I}}$ has to be specified as well.

For the design of a CM in the Case (3), the output displacement is desired to achieve $X_{\mathrm{O}}^{*}$. Design problems are stated as follows:

- DPG: given $P_{\mathrm{I}}, P_{\mathrm{O}}$ and $\left[\mathbf{K}_{\mathrm{A}}\right]$, design a $\mathrm{CM}$ so that its $X_{\mathrm{O}}$ can achieve $X_{\mathrm{O}}^{*}$.

- DPH: given $X_{\mathrm{I}}, P_{\mathrm{O}}$ and $\left[\mathbf{K}_{\mathrm{A}}\right]$, design a CM so that its $X_{\mathrm{O}}$ can achieve $X_{\mathrm{O}}^{*}$.

Note that any one or two or three of the $P_{\mathrm{I}}, X_{\mathrm{I}}$ and $\left[\mathbf{K}_{\mathrm{A}}\right]$ could also be unknown (unknowns) and need to be optimally determined. Be noticed that, by optimally determining $\left[\mathbf{K}_{\mathrm{A}}\right]$, the global stiffness matrix of the actuator, we actually determine the local stiffness matrix of the actuator and the location of the actuator.

\subsection{Case (4)}

- DPI: given $X_{\mathrm{I}}$ and $\left[\mathbf{K}_{\mathrm{A}}\right]$ and $\left[\mathbf{K}_{\mathrm{W}}\right]$, design a CM so that its $X_{\mathrm{O}}$ can achieve $X_{\mathrm{O}}^{*}$.

- DPJ: given $P_{\mathrm{I}}$ and $\left[\mathbf{K}_{\mathrm{A}}\right]$ and $\left[\mathbf{K}_{\mathrm{W}}\right]$, design a CM so that its $X_{\mathrm{O}}$ can achieve $X_{\mathrm{O}}^{*}$.

- DPK: given $X_{\mathrm{I}}$ and $\left[\mathbf{K}_{\mathrm{A}}\right]$ and $\left[\mathbf{K}_{\mathrm{W}}\right]$, design a CM so that its $P_{\mathrm{O}}$ can achieve $P_{\mathrm{O}}^{*}$.

- DPL: given $P_{\mathrm{I}}$ and $\left[\mathbf{K}_{\mathrm{A}}\right]$ and $\left[\mathbf{K}_{\mathrm{W}}\right]$, design a CM so that its $P_{\mathrm{O}}$ can achieve $P_{\mathrm{O}}^{*}$.

Note that any one or two or three or four of the $P_{\mathrm{I}}, X_{\mathrm{I}},\left[\mathbf{K}_{\mathrm{A}}\right]$ and $\left[\mathbf{K}_{\mathrm{W}}\right]$ could also be unknown and need to be optimally determined.

The above design problems are general in the sense that they are not explicitly tied with mechanism design problems, i.e. FG, PG and MG. Note that these three design problems are typical for the design of rigid-body mechanisms. For the design of CMs, they need to be modified to fit into the abovedefined general design problems from DPA to DPL.

FG for rigid-body mechanisms is defined as the correlation of an input motion with an output motion in a mechanism (Norton, 2003). A function generator is conceptually a black box that, for a given value of input motion, an output value of motion is also specified through a function which relates input motions and output motions. For example, in a rigid four-bar mechanism, the function shows the relationship between rotations of input link and rotations of output crank. While it comes to CMs, the FG is the correlation of the input with the output in the context of deformable object, which means that not only displacements but also forces can be one of the correlated problem state variables. For example, for a CM for FG designed from DPB, the output displacements of the $\mathrm{CM}$ are correlated with the input forces. All design problems from DPA to DPL can be extended for the design of CMs for FG by simply considering a function between inputs and outputs. There are many potential applications of CMs for FG, e.g. displacement amplifiers for PZT actuators (Canfield and Frecker, 2000), optical modulating component modulator (driven by comb actuators) which needs a planar angular rotator to control different angles and to modulate the dissimilar light (Lin and Shih, 2002).

PG is defined as the control of a point on a mechanism such that it follows a prescribed path (Norton, 2003). The position of this point can either be correlated to the input motion or not. Since the point on a mechanism cannot completely follow the prescribed path, some precision points on the path are selected so that the point on the mechanism can approximately follow the path by going through all the precision points. Design problems of DPA, DPB, DPC, DPD, DPG, DPH, DPI, DPJ, DPH and DPI can be extended for the design of CMs for PG by simply considering a sequence of different values of output displacements, i.e. $X_{\mathrm{O}}$, to represent precision points. If the correlation between inputs and 
Initial configuration

A

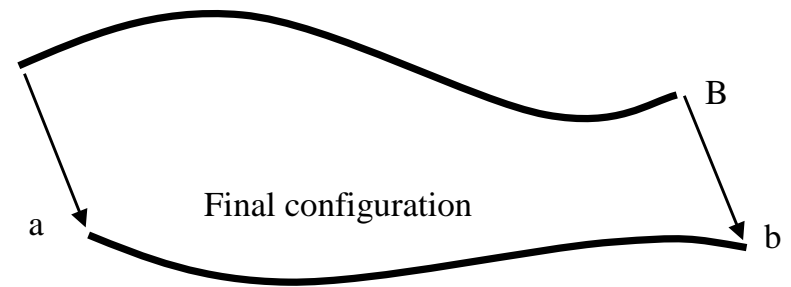

Figure 3. Compliant-segment motion generation (Saggere and Kota, 2001).

outputs is not required, then the inputs can be optimally determined as well.

MG is defined as guiding an entire (rigid) body, which is usually a part of a rigid floating link, through a prescribed motion sequence, which comprises of desired positions and orientations of the floating link. However, if the body to be guided is compliant (flexible), its deformation should be counted into the position changes. Thus, the task of a CM for MG is to guide a given slender flexible segment to different desired configurations. That is, the given flexible segment is to be deformed into another specified definite smooth shape while moving it from its initial configuration to another specified configuration as illustrated in Fig. 3. Such a task is called "compliant-segment motion generation" task (Saggere and Kota, 2001). In this paper hereafter we use "MG" to represent "compliant-segment motion generation" for short. Thus, MG is to guide a flexible segment of a mechanism through a sequence of discrete prescribed configurations. The configurations can either be correlated to the input motion or not. By defining the configuration changes as the output displacements $X_{\mathrm{O}}$, design problems of DPA, DPB, DPC, DPD, DPG, DPH, DPI, DPJ, DPH and DPI can be extended for the design of CMs for MG. If the correlation between inputs and outputs is not required, then the inputs can be optimally determined as well.

The above discussion is applicable to both planar and spatial CMs. The next section gives a critical review of the literature on the design of CMs through the TO technique.

\section{Critical review of the literature}

Two key aspects of CM design through the TO technique are: (1) the working conditions or boundary conditions, particularly force boundary conditions and displacement boundary conditions; (2) the design purposes, i.e. functional requirements. Boundary conditions determine how a CM interacts with its environments, i.e. the support regions, actuators and load objects, and they determine the working conditions of a CM, particularly force or displacement conditions at the input port and output port of the CM (the support region also make a great difference). The functional requirements depend on applications, and they determine how the $\mathrm{CM}$ is expected to response under given boundary conditions.

Design specifications that reveal working conditions of a $\mathrm{CM}$ are defined in the previous section of the paper, and these working conditions are reviewed in the first part of the literature review section. Functional requirements from the perspective of mechanism design are defined in the previous section, and functional requirements considered in the literature are also reviewed in the second part through discussions of the formulations that have been built. The third part discusses about the point-flexure problem in the TO of CMs.

\subsection{Boundary conditions}

Many studies have, incompletely or with some differences, designed CMs by considering the work conditions in the design specification (1), (2), (3) or (4).

Ananthasuresh (1994) specified the input force $P_{\mathrm{I}}$ and output force $P_{\mathrm{O}}$ in a multi-criteria model. In the model, two loadings were considered separately. First, a CM was deformed to contact with a stiff work-piece under the input force $P_{\mathrm{I}}$. Second, as a force of resistance, the work-piece applied output force $P_{\mathrm{O}}$ to the CM; meanwhile, the input force increased to $P_{\mathrm{I}}+\Delta P_{\mathrm{I}}$. The output displacement $X_{\mathrm{O}}$ was considered only from the first loading, i.e. the input force $P_{\mathrm{I}}$. Although the loads described in the model were the same with those in the DPB of the case (1), the loadings were different from the real situation because the output force $P_{\mathrm{O}}$ had influence on the $X_{\mathrm{O}}$ and the $P_{\mathrm{O}}$ should be considered into the calculation of $X_{\mathrm{O}}$. Frecker et al. (1997) and Kikuchi et al. (1998) also considered the same loadings in their designs. Hetrick (1999) also used two loadings, though, in a different way. First, the output force $P_{\mathrm{O}}$ was applied while the input port was held fixed, then the actuator generated an input force to actuate the input port so that the output port moved in the desired direction. The loads described in the model were the same with those in the DPB of the case (1), and the calculated output deformation included the influence from the $P_{\mathrm{O}}$. Designs based on these approaches are highly dependent on the output forces. Moreover, the loads of a CM are usually separated into different loading steps. This is appropriate, most of the time, for the design of CMs in the linear force-deflection range, however, may not be appropriate in the non-linear force-deflection range.

To account for the stiffness of elastic work-pieces, i.e. the case (2), a so-called spring model have been developed (Ananthasuresh, 1994; Saxena and Ananthasuresh, 2000; Deepak et al., 2009). In the model, the work-piece was modeled as a spring of constant stiffness, i.e. given $\left[\mathbf{K}_{\mathrm{W}}\right]$. However, most studies have specified input force, as stated in the DPD and DPF, but not input displacements, as stated in the DPC and DPE. One exception is the study by Joo and Kota (2004). They specified, at stated in the DPC and DPE, the input displacement $X_{\mathrm{I}}$ and the stiffness of the spring at the output port. Huang and Lan (2006) explored the influence of 
different stiffness values of springs at output port on the optimal topologies. Their research shows that the stiffness of the spring makes a great difference to the results.

Regarding the way a CM interacts with a work-piece, Sigmund (1997) considered three different loading situations: (1) the work-piece was stiff and there was no gap between the work-piece and the output port; (2) the work-piece was elastic and there was no gap; (3) the work-piece was elastic and there is a gap. The first situation, in fact, is not a loading case of a mechanism since there is no displacement at the output port. In the second and third situations, the input force $P_{\mathrm{I}}$ and the $\left[\mathbf{K}_{\mathrm{W}}\right]$, the same as that in the DPD, were specified. The third situation can be understood as the nonlinear behaviour of the work-piece. Saxena and Ananthasuresh (2001) designed CMs for path generation, specifying a sequence of input forces, $P_{\mathrm{I} 1}, P_{\mathrm{I} 2}$, etc., while two linear springs of constant stiffness along two orthogonal directions were used to model the work-pieces. This is an extension of the DPD for the path generation problem. However, they did not consider the coupling between the two linear springs and the nonlinear behaviour of work-pieces.

To account for the stiffness of both the actuator and workpiece, i.e. the same as those in the DPJ and DPL in the case (4), Du et al. (2000) modeled a actuator as a rod element (stiffness is $\left[\mathbf{K}_{\mathrm{A}}\right]$ ) and a force $P_{\mathrm{I}}$; on the other hand, the work-piece was modeled as a linear spring (stiffness is $\left[\mathbf{K}_{\mathrm{W}}\right]$ ). Luo et al. (2005) also considered the same problem.

Pedersen et al. (2001) considered path generation, specifying a sequence of input displacements, $X_{\mathrm{I} 1}, X_{\mathrm{I} 2}$, ect., while output forces, $P_{\mathrm{O} 1}, P_{\mathrm{O} 2}$, etc., were considered at precision points. Tai et al. (2002) also considered path generation, specifying input displacements, $X_{\mathrm{I} 1}, X_{\mathrm{I} 2}$, ect., while the output forces were specified as zeros. Both studies are the extensions of the DPA for the path generation problem. Rai et al. (2007) considered path generation, specifying a sequence of input forces, $P_{\mathrm{I} 1}, P_{\mathrm{I} 2}$, etc., while the output force were specified as zeros. This is an extension of the DPB for the path generation problem.

Regardless of the functional requirements of CMs, there is no universal boundary condition for the design CMs in the literature. Certainly, the loading cases (1), (2) and (4) have been considered, at least to some degree. However, it is important to note that they are still incomplete and the loading case (3) has yet to be considered.

To account for the work-piece at the output of a CM, a spring has been added to the output port to model the behaviour of the work-piece (Sigmund, 1997; Ananthasuresh, 1994). To account for both the actuator and the work-piece, a spring is added to each port (Du et al., 2000; Luo et al., 2005; Huang and Lan, 2006; Sigmund, 2002). The spring model, which is widely accepted in the literature, captures the behaviour of the actuator and work-piece. However, optimal results are highly dependent on the stiffness of springs (Wang, 2009b; Luo et al., 2005) so that the optimal CMs can only work properly with certain actuators or work-pieces which have the same stiffness.

Translational motions or forces have been considered in the literature, however, rotational motions or torques (Eqs. 1 and 2) have not yet while rotational inputs or outputs are essential and common in applications, e.g. optical modulating component modulator (driven by a comb actuator). The modulator needs a planar angular rotator to control different angles and to modulate the dissimilar light (Lin and Shih, 2002).

Future work regarding boundary conditions lies in: (1) the accurate modeling of work-pieces in the case of path generation and motion generation CMs; (2) the design of CMs that are not sensitive to the stiffness of the work-piece or actuators; (3) the design of CMs with variable boundary conditions, e.g. input forces or support regions are design variable; (4) the consideration of rotational inputs and outputs. (5) the design of CMs with specified actuators and output forces, i.e. the design specification (3).

\subsection{Formulations for functional requirements}

Although various functional requirements have been considered in the design of CMs (e.g. inverters, crimpers and grippers), the design problems can be generalized into two kinds: qualitative and quantitative design problems. In the first group, functional requirements include maximized or minimized mechanical/geometric advantage, work ratio, etc., and there is no direct quantitative control over the magnitudes of these performance; in the second group, the functional requirements includes output displacements, geometric advantage, are required to achieve exact values instead of just being optimized for extreme values. A variety of formulations (Table 2) from either the viewpoint of structure or mechanism design have been built to address these design problems. Some of these formulations have been developed to avoid the flexure-point problem, a very common problem in most formulations.

When designing CMs for maximized or minimized characteristics, F1-F14 are the most common formulations encountered; thus, the design problems are classified as CM qualitative design problems. In contrast, when designing CMs for direct quantitative control over the magnitudes of characteristics, F15-F21 are the most common formulations encountered; thus, these design problems are classified as CM qualitative design problems. When designing CMs for distributed compliance or the removal of point flexures, F22F27 are the most common problems encountered; certainly, these design problems fall into either the qualitative design of CMs or the quantitative design of CMs, but with special attention to the generation of point flexures. 
Table 2. Formulations in the Literature.

\begin{tabular}{|c|c|c|c|}
\hline References & $\begin{array}{l}\text { Functional } \\
\text { Requirements }\end{array}$ & Formulations & $\begin{array}{l}\text { Specified inputs/ } \\
\text { outputs }\end{array}$ \\
\hline \multicolumn{4}{|l|}{ Formulations for quantitative design of $\mathrm{CMs}$} \\
\hline F1 (Ananthasuresh, 1994) & Flexibility-stiffness & Max: $w_{1} \cdot \operatorname{MSE}-\left(1-w_{1}\right) \cdot \mathrm{SE}$ & $P_{\mathrm{I}}$ and $P_{\mathrm{O}}$ \\
\hline $\begin{array}{l}\text { F2 (Frecker et al., 1997; Huang and Lan, } \\
\text { 2006; Deepak et al., 2009) }\end{array}$ & Flexibility-stiffness & Max: MSE/SEO & $P_{\mathrm{I}}$ and $P_{\mathrm{O}}$ \\
\hline F3 (Nishiwaki et al., 2001) & Flexibility-stiffness & Max: $\frac{\text { MSE }}{w_{1} \cdot X_{\mathrm{O}-\mathrm{P}+w_{2} \cdot \operatorname{SEI}+\left(1-w_{1}-w_{2}\right) \cdot \mathrm{SEO}}}$ & $P_{\mathrm{I}}$ and $P_{\mathrm{O}}$ \\
\hline F4 (Joo et al., 2000) & Flexibility-stiffness & Max: $\frac{\text { MSE }}{w_{1} \cdot \operatorname{SEI}+\left(1-w_{1}\right) \cdot S E O}$ & $X_{\mathrm{I}}$ and $P_{\mathrm{O}}$ \\
\hline F5 (Saxena and Ananthasuresh, 2000) & Flexibility-stiffness & $\begin{array}{l}\text { Min: }-\eta(\mathrm{MSE}) / \mu(\mathrm{SEI}), \text { where } \\
\frac{\partial \eta\left(X_{\mathrm{O}}\right)}{\partial X_{\mathrm{O}}}>0 ; \frac{\partial g(\mathrm{SEI})}{\partial \mathrm{SEI}}>0\end{array}$ & $\begin{array}{l}P_{\mathrm{I}} \text { and } \\
P_{\mathrm{O}}=k_{\mathrm{O}} \cdot X_{\mathrm{O}}\end{array}$ \\
\hline F6 (Saxena and Ananthasuresh, 1998) & Flexibility-stiffness & Max: $\frac{\text { outputenergy }}{\mathrm{SEI}}$ & $\begin{array}{l}P_{\mathrm{I}} \text { and } \\
P_{\mathrm{O}}=k_{\mathrm{O}} \cdot X_{\mathrm{O}}\end{array}$ \\
\hline F7 (Kota et al., 2001) & Flexibility-stiffness & Max: $\frac{\mathrm{GA}}{\mathrm{SE}}$ & \\
\hline F8 (Ananthasuresh, 1994) & Flexibility-stiffness & Min: SE & $\begin{array}{l}P_{\mathrm{I}} \text { and } \\
P_{\mathrm{O}}=k_{\mathrm{O}} \cdot X_{\mathrm{O}}\end{array}$ \\
\hline F9 (Bendsoe and Sigmund, 2004) & Output displacement & Max: $X_{\mathrm{O}}$ & $\begin{array}{l}P_{\mathrm{I}}, P_{\mathrm{I}}=g\left(X_{\mathrm{I}}\right) \text { and } \\
P_{\mathrm{O}}=f\left(X_{\mathrm{O}}\right)\end{array}$ \\
\hline $\begin{array}{l}\text { F10 (Sigmund, 1997; Lau et al., 2001; Wang, } \\
\text { 2009c; Deepak et al., 2009) }\end{array}$ & MA & Max: MA, subjecting to $X_{\mathrm{I}} \leq X_{\mathrm{I}}^{*}$ & $\begin{array}{l}P_{\mathrm{I}} \text { and } \\
P_{\mathrm{O}}=f\left(X_{\mathrm{O}}\right)\end{array}$ \\
\hline F11 (Lau et al., 2001) & GA & Max: GA, subjecting to $X_{\mathrm{O}} \leq X_{\mathrm{O}}^{*}$ & $P_{\mathrm{I}}$ and $P_{\mathrm{O}}=k_{\mathrm{s}} \cdot X_{\mathrm{O}}$ \\
\hline F12 (Lau et al., 2001) & ME & Max: ME, subject to $X_{\mathrm{O}} \leq X_{\mathrm{O}}^{*}$ & $P_{\mathrm{I}}$ and $P_{\mathrm{O}}=k_{\mathrm{S}} \cdot X_{\mathrm{O}}$ \\
\hline F13 (Hetrick, 1999; Deepak et al., 2009) & ME & Max: ME & $P_{\mathrm{I}}$ and $P_{\mathrm{O}}$ \\
\hline F14 (Canfield and Frecker, 2000) & ME & Max: ME & $P_{\mathrm{I}}$ and $P_{\mathrm{O}}=k_{\mathrm{s}} \cdot X_{\mathrm{O}}$ \\
\hline \multicolumn{4}{|l|}{ Formulations for quantitative design of $\mathrm{CMs}$} \\
\hline F15 (Min and Kim, 2004) & $\mathrm{GA}^{*}$ & $\begin{array}{l}\text { Min: } w_{1} \cdot\left(\mathrm{GA}^{*}-\frac{X_{\mathrm{O}}}{X_{\mathrm{I}}}\right)^{2}+\left(1-w_{1}\right)\left(w_{2} .\right. \\
\left.\mathrm{SEI}+\left(1-w_{2}\right) \cdot \mathrm{SEO}\right)\end{array}$ & $P_{\mathrm{I}}$ and $P_{\mathrm{O}}$ \\
\hline F16 (Pedersen et al., 2001) & PG & Min: LSE1 & $X_{\mathrm{I}}$ and $P_{\mathrm{O}}$ \\
\hline F17 (Saxena and Ananthasuresh, 2001) & PG & Min: LSE2 & $X_{\mathrm{I}}$ and $P_{\mathrm{O}}=k_{\mathrm{s}} \cdot X_{\mathrm{O}}$ \\
\hline F18 (Tai et al., 2002) & PG & Min: LSE3 & $X_{\mathrm{I}}$ and $P_{\mathrm{O}}$ \\
\hline F19 (Saxena, 2005) & PG & Min: LSE4 & $P_{\mathrm{I}}$ and $P_{\mathrm{O}}$ \\
\hline F20 (Rai et al., 2007, 2009) & PG & Min: $\sum w_{i} \operatorname{err}_{i}$ & $P_{\mathrm{I}}$ and $P_{\mathrm{O}}$ \\
\hline \multicolumn{4}{|l|}{ Formulations for point flexure problem } \\
\hline F21 (Lee, 2011) & Flexibility-stiffness & Max: $w_{1} \cdot \operatorname{MSE}-\left(1-w_{1}\right) \cdot \sum \varepsilon_{i}^{2}$ & $P_{\mathrm{I}}$ and $P_{\mathrm{O}}$ \\
\hline F22 (Yin and Ananthasuresh, 2003) & Output displacement & Min: $-\frac{\text { MSE }}{\Phi}$ & $P_{\mathrm{I}}$ and $P_{\mathrm{O}}$ \\
\hline $\begin{array}{l}\text { F23 (Deepak et al., 2009; Rahmatalla and } \\
\text { Swan, 2005) }\end{array}$ & Output displacement & $\begin{array}{l}\text { Max: } X_{\mathrm{O} 1} \\
\text { subjecting to } X^{*} \leq X_{\mathrm{I} 2}\end{array}$ & $P_{\mathrm{I}}$ and $P_{\mathrm{O}}=k_{\mathrm{s}} \cdot X_{\mathrm{O}}$ \\
\hline F24 (Cardoso and Fonseca, 2004) & Flexibility-stiffness & Max: SE & $P_{\mathrm{I}}$ and $P_{\mathrm{O}}=k_{\mathrm{s}} \cdot X_{\mathrm{O}}$ \\
\hline $\begin{array}{l}\text { F25 (Deepak et al., 2009; } \\
\text { Chen and Wang, 2007) }\end{array}$ & GA* & Max: $e^{-\left(\mathrm{GA}-\mathrm{GA}^{*}\right) 2} \mathbf{K}_{11} \mathbf{K}_{22}$ & $P_{\mathrm{I}}$ and $P_{\mathrm{O}}$ \\
\hline
\end{tabular}

MSE: Mutual Strain Energy; SE: Strain Energy due to $P_{\mathrm{I}}$ and $P_{\mathrm{O}}$, SEO: Strain Energy due to the output force $P_{\mathrm{O}}$ with the input port and the support region being fixed, SEI: Strain Energy due to input force $P_{\mathrm{I}}$ with the output port and the support region being fixed, $w_{1}, w_{2}, \ldots, w_{i}$ : weighting factors, $X_{\mathrm{O}-\mathrm{P}}$ : the displacement of the output port perpendicular to the desired direction, err ${ }_{i}$ : Fourier coefficients errors between the desired and actual paths, $\varepsilon_{i}$ : the strain due to input forces and output forces, $\Phi$ : the sum of local relative rotations, $X_{\mathrm{O} 1}$ : output displacement when two springs of high stiffness are connected both at the input and at the output, $X_{\mathrm{I} 2}$ : output displacement when only a spring of moderate stiffness is connected to the output, $\mathbf{K}_{11}, \mathbf{K}_{22}$ : the condensed stiffness matrices, LSE1: $\min \Phi=\sum_{j=0}^{2} \alpha_{j} \sum_{i=1}^{M}\left[\Delta_{\text {out }, i, j}-\Delta_{\text {out }, i}^{*}\right]^{2} ; \operatorname{LSE} 2$ :

$\Phi=\sum_{i=1}^{M}\left[\left(\delta X_{\mathrm{I}}-\delta X_{\mathrm{I}}^{*}\right)^{2}+\left(\delta y_{i}-\delta y_{i}^{*}\right)^{2}\right]$, LSE3: $\Phi=\frac{1}{M} \sum_{i=1}^{M}\left[\left(\delta X_{\mathrm{I}}-\delta X_{\mathrm{I}}^{*}\right)^{2}+\left(\delta y_{i}-\delta y_{i}^{*}\right)^{2}\right]^{1 / 2}$, LSE4: $\Phi=\left(Q_{i}-P_{\mathrm{I}}\right) \cdot\left(Q_{i}-P_{\mathrm{I}}\right)$, where $Q_{i}$ denotes the actual deformation of the output port for the actuation forces $F_{i}$ while $P_{\mathrm{I}}$ is the desired deformation, $i=1,2,3, \ldots, n ; n$ is number of precision points (the un-deformed output position is not included). 


\subsubsection{Formulations for qualitative design of CMs: F1-F14}

Within F1-F14, F1-F8 are for the purpose of flexibilitystiffness, while F9-F14 are for mechanical functional requirements, e.g. MA, GA, etc. In F1-F8, the strain energy (SE), a concept commonly used in the structural optimization, is employed. Moreover, these formulations become a trend to design CMs for the so-called flexibilitystiffness purpose: (a) flexibility to undergo desired deformations (kinematic requirement); (b) Stiffness to bear external loads (structural requirement). Hence, multi-criteria formulations are built to design $\mathrm{CMs}$ so that the $\mathrm{CMs}$ are flexible enough to deform and stiff enough to resist loads. The flexibility is always formulated as Mutual Strain Energy (F1, F2, F3, and F4) which numerically equals output deformation. Other formulations for flexibility include a variant of MSE (F5), or mechanical properties, e.g. output energy (F6, F8), GA (F7). Since stiffness, on the other hand, represents the compliance of a structure, so stiffness is formulated as SE, which is essentially the compliance of a structure. The lower the strain energy, the stiffer the structure. It is preferable to formulate these criteria in the form of a ratio (F2-F5) than to formulate them in the form of a summation (F1). With the form of a ratio of these criteria, there is no need to select weighting factors, and the multiple criteria have the same importance in the objective function so that none of them will appear to dominate the combined objective function.

Regarding these multi-criteria formulations (F1-F7), researchers argued that if there was no structural requirement, the optimal results tended to be infinitely flexible and connections among points of interest could not be assured. However, this is not true for the following reasons: first, from the viewpoint of the design problems of CMs, stiffness is not even a primary requirement for a mechanism; therefore, it is not reasonable to consider it as the primary requirement in formulations, e.g. output forces, output displacement. Second, the reason why connections are not ensured is that the employed optimization algorithms cannot avoid topologically disconnected topologies. However, connected topologies can actually be guaranteed by improving the ability of algorithms instead of considering stiffness in formulations. Lu and Kota (2003) developed a representation of load path scheme to avoid topological disconnected structures. The scheme excludes topological disconnected structures at the beginning of optimization. Zhou and Ting (2005) introduced spanning tree theory to weed out invalid disconnected topologies. Third, maximizing stiffness, either explicitly or implicitly, leads to lumped CMs, where point flexures appear, so that these CMs cannot be manufactured and function well in practical use. The point flexure problem is discussed further in the next section. Fourth, infinitely flexible topologies can be avoided by considering constraints on input or output displacements, stress, etc. To sum up, stiffness should not be as important as functional requirements as a criterion of mechanisms, e.g. output forces and output displacements, in the design of CMs.

Ananthasuresh (1994) designed CMs by minimizing SE (F8) under the case of spring model, where SE equals the difference of input energy and energy stored in the spring. The spring model captures the feature of the work-piece the mechanism works with. The stiffness of the spring is chosen depending on the stiffness of the work-piece, a hard workpiece can be modeled as a spring with high stiffness and vice versa. Minimizing SE is actually to find a balance of input energy (minimizing input energy) and energy stored in the spring (maximizing energy stored in the spring). The input energy is determined by the input force and input displacement while the energy stored in the spring is determined by deformation of the spring (or the output displacement) and the stiffness of the spring. Comparing with F1-F7, F8 is compact and it gives more reasonable results. However, in F8, a very careful choice of input force and stiffness of the spring is required to get reasonable optimal topology. Both of the input force and the stiffness of the spring need to match each other so that neither of them dominates the value of SE. Nevertheless, in the practical use of CMs, there is no much space for designers to pick input forces and work-pieces. Further, the output displacement is only implicitly included in the objective function while mechanisms are always designed for explicit displacement requirements.

In F9-F14, instead of being designed for structural properties, i.e. maximized strain energy, CMs are designed purely for mechanical functional requirements, e.g. output displacement (F9), MA (F10), GA (F11) ME (F12-F14). Sigmund (2002) designed displacement amplifiers by maximizing the output displacement (F9). Both the actuator and work-piece were modeled as springs. Sigmund (1997) formulated MA (F10) as objective function to design CMs and two loading conditions were considered, i.e. with gap or without gap between the $\mathrm{CM}$ and the work-piece. Lau et al. (2001) considered MA (F10), GA (F11) and ME (F12) as objective functions, respectively. Resistance force due to work-piece was considered using the spring model. The properties of obtained topologies were consistent with the employed objective functions from the viewpoint of mechanical performances. By considering formulations for mechanical functional requirements in the design of CMs, instead of getting the same results with those for flexibility-stiffness purpose, optimal topologies, whose performances are consistent with the mechanical functional requirements, can be obtained. However, there are nonlinear constraints in all the formulations for MA and GA, which brings difficulty in algorithm convergence and the results suffer from point flexure problem (Deepak et al., 2009; Wang, 2009b).

Canfield and Frecker (2000) designed compliant displacement amplifier for stack actuators by maximizing ME (F14), which was the product of GA and MA. GA is measured under the free displacement condition and the MA is measured under the blocked force condition, and these conditions 

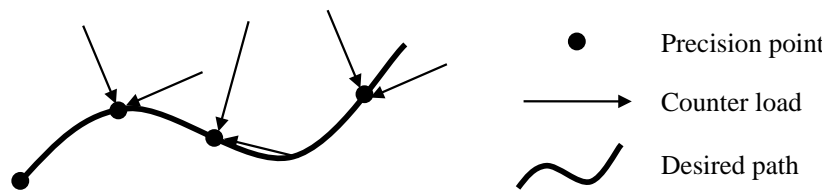

Figure 4. Path with counter loads (Pedersen et al., 2001).

cannot be simultaneously obtained, the defined ME does not exactly capture the real mechanical efficiency of the system and it only represents a theoretical maximum (Frecker and Canfield, 2000). Moreover, even though the spring model is considered for the work-piece, the resistance force from the work-piece is not included into the kinematic analysis and it is only for the calculation of output energy which is stored in the spring.

For formulations regarding ME, it is not necessary to add any kinematic constraints, since ME is naturally constrained to be less than one. From this point of view, it is more convenient to implement the optimization problem of the ME formulations (F13, F14). However, it is mainly dependent on the design problems regarding which formulation to select.

Besides the above discussion regarding these formulations, several problems need to be pointed out:

First, the results are all sensitive to the output loads, i.e. the magnitude of output forces, the stiffness of springs at the output port. Second, the results suffer from point flexure problem and it is essentially common in all formulations. Third, CMs are designed in a qualitative sense instead of a quantitative sense. However, according to the discussions on DPA to DPN, CMs are always required to generate exact magnitude of displacements or forces. Fourth, most formulations specify input forces rather than input displacements. However, according to the previously defined design problems (DPADPN), not only forces, but also motions could be specified as inputs of a mechanism. In addition, specifying displacements as inputs have several benefits, i.e. the solutions are less sensitive to the lower bounds of design variables. Last but not the least, inputs could also be set design variables instead of being specified since in the case of large deformation, specifying inputs may result in the missing of possible optimal topologies of CMs which can generate the motion required under the actuation of other inputs. This point also fits to the mechanism kinematic design problem, i.e. in PG without prescribed timing and MG without prescribed timing design problem, only the output motions are concerned. Fifth, only translational inputs are considered in these formulations, however, rotational motions or forces are not considered (Eqs. 1 and 2) while rotational inputs or outputs are essential and common in applications, i.e. optical modulating component modulator (driven by a comb actuator) which needs a planar angular rotator to control different angles and to modulate the dissimilar light (Lin and Shih, 2002).

\subsubsection{Formulations for quantitative design of CMs: F15-F21}

Min and Kim (2004) designed CMs for specified magnitude of GA (F15) so that GA could be controlled directly and quantitatively. F16-F20 are formulations to design CMs for path generation based on the previously defined DPB or DPF. Pedersen et al. (2001) design path-following CMs (F16) so that the output port passes the precision points due to corresponding input displacements, and it also passes the same precision points when two separate counter loads (output forces) are applied at each precision points. One is against the output direction and one is perpendicular to the output direction (Fig. 4). The input forces are constrained to upper limits. A weighted sum of Least Square Errors (LSE), which is the difference between the desired output displacements and the obtained output displacement, is formulated for the synthesis of CMs for PG.

Including the counter loads in this model makes the optimal mechanism stiff enough to resist the counter loads. However the counter load in this model is selected based on the input/output displacement and the actuator, which is not true. In reality, the counter load is dependent on the work-piece the mechanism works with, instead of actuators. In addition, this model only guarantees the deformation in the desired direction, the deformation in its perpendicular direction is not concerned so that the output port may not pass through the precision points even though the error function is very close to zero.

Saxena and Ananthasuresh (2001) designs CMs for path generation (F17) with a sum of least square errors (LSE2) in a $x-y$ coordinate system while the work-piece the CM works with are modeled as linear springs. Precision points in the design domain are described with an $x-y$ coordinates so that the output port needs to traverse through points $\mathrm{P}_{i}$ of coordinates $\left(\delta X_{\mathrm{I}}^{*}, \delta y_{i}^{*}\right)$ with respect to its undeformed position due to input forces. The work piece at the output port is modeled as two linear springs. Here, two linear springs are added along the $x$ and $y$ displacement directions at the output port to simulate the resistance along the linear path. However, like all other models based on the spring model, the optimal results highly depend on the stiffness of the two springs. Moreover, the spring model cannot precisely capture the complicated interaction between the CM and the work-piece in the case of path generation.

Tai et al. (2002) designed path following CMs (F18) so that the output port passed a sequence of precision points on a path with specified input displacements and specified output forces (output forces are specified as zero). The magnitudes of the input displacements were given, and the forces that needed to generate the input displacements were constrained to an upper limit. However, the output loads were specified as zero so that the connections cannot be guaranteed if no other filter were employed. 


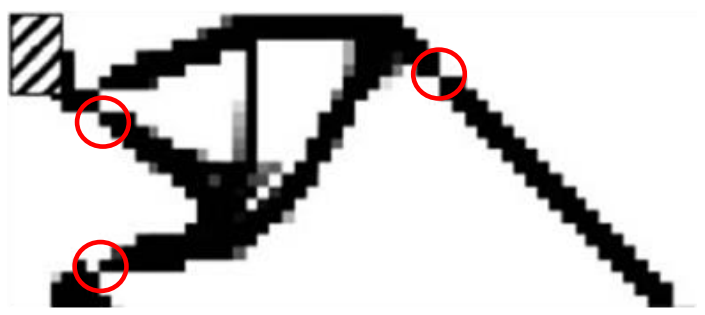

Figure 5. An optimal topology for a compliant gripper from F13 (Deepak et al., 2009).

Saxena (2005) designed path following CMs (F19) so that the output port passed a sequence of precision points on a path with specified input forces and specified stiffness of spring at the output port. There were $\mathrm{n}$ objectives functions for $\mathrm{n}$ precision points. The multiple objectives were minimized simultaneously, and a set of Pareto optimal solutions among were obtained. In these solutions, a solution that corresponds to the minimum of each objective existed, and the solution of the combined summation of all the individual objective functions also existed. This idea may provide designers more choices for desired solution from these Pareto optimal ones. But results showed that all the optimal results could only capture the trend of the specified path, but could not be desirably close to the specified path.

The optimal synthesis of mechanisms for path generation commonly minimizes the sum of error functions, taking the mean squared distance between the obtained curve and the desired curve over a number of precision points as the structural error. The error function attempts to compare the shape, size, orientation, and location of a desired curve with an actually obtained curve all at once thereby simultaneously limiting the search space and making the search intractable.

Besides the LSE functions, Fourier Descriptors method (Ullah and Kota, 1997) was employed to formulate the objective function (F20) for the path following CMs optimal synthesis by Rai et al. (2007). The Fourier Descriptors objective function compares purely the shape of two plane curves without being affected by the location, size, or orientation differences between curves. If the shape of the actual obtained curve is the same as the desired one, by translating, rotating and scaling the solution mechanism appropriately, without changing topology of the mechanism, the solution curve can be made to coincide with the desired curve in shape, position, orientation and size. Apparently this may make the design non-systematic since human interruptions are required to change the design to meet the function requirements.

We conclude that there are mainly two types of formulations for the design of path-following CMs. One is the formulations based on the LSE and the other is based on the Fourier Descriptors. Although there are different forms of LSE functions, the essence in these forms is the same, i.e. the coordinate differences of precision points on the path. With the LSE formulation, one can design CMs for any kinds

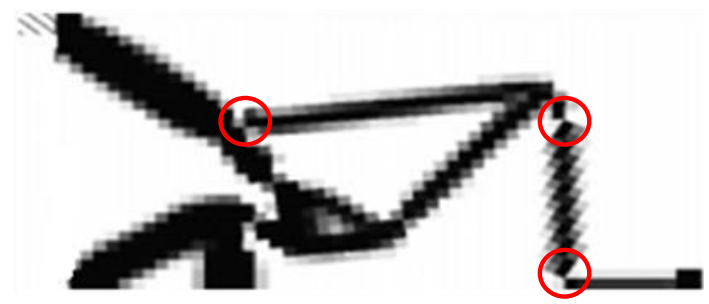

Figure 6. An optimal topology for a path-following CM from F16 (Pederson et al., 2001).

of output displacement requirements or any kinds of inputoutput relationships. For example, one can design CMs for linear or nonlinear input-output relationship (Pedersen et al., 2001; Saxena and Ananthasuresh, 2001); one can also design CMs to follow linear or nonlinear paths (Pedersen et al., 2001; Saxena, 2005). This is very essential since designers can directly, precisely and quantitatively control the inputs and outputs; while F1-F14 are only for the qualitative design CMs. In contrast, with the formulations based on Fourier Descriptors, one can only design a CM to follow the shape of a path; thus, this approach is only useful when the shape of a path is the concern.

In addition, formulations for the design of path-following CMs also have the same problems as stated as the first, second and fifth points in previous part of this section.

\subsection{Formulations for point flexure problem}

A common problem in the above mentioned formulations is the presence of point flexures, i.e. two solid elements connect to each other diagonally by one node, as shown in the circled regions in Figs. 5 and 6. Point flexures undesirable in CMs since it is impossible to manufacture them, and replacements have to be employed (Yin and Ananthasuresh, 2003). Moreover, even replacements are used, they still suffer high stress, which leads to yield failure, or fatigue failure. The presence of point flexures leads to a lumped compliant system whose compliance is concentrated on several local regions as opposed to the desired one called distributed compliant mechanism whose compliance is distributed evenly to the whole body of the CM. There are methods taking efforts to design CMs without point flexures by considering alternative parameterization methods or filters; however, only methods on alternative formulations are discussed in the paper.

Yin and Ananthasuresh (2003) proposed a method to restrain local, relative rotation with a novel that hopes to make the local deformation uniform throughout the structure. It is explained that the optimization algorithm tends to generate point flexures since they undergo for large displacements without the cost of high strain energy, both of which are just what are desired in the formulations. For example, in F2, the ratio of output displacement to the strain energy is maximized, point flexures is just the ideal component that 
can maximize the ratio. With the relative rotation being restrained, the formulation (F22) is able to remove point flexures and give distributed compliant designs. However, this approach only restrains the relative rotations, which actually limits the degree of lumped compliance but it does not touch the essence of the problem, i.e. it is the formulation that prefers to topologies with minimum strain energy in a $\mathrm{CM}$ and maximum displacement at the output port, which leads to the tendency of lumped compliance, even point flexures. Restricting the relative rotation can only prevent the relative rotation but it cannot change the tendency of lumped compliance.

With the similar explanation for the point flexure problem, Cardoso and Fonseca (2004) stated that strain energy should not be minimized since a distributed compliant mechanism must spend a part of the input energy in the form of strain energy to deform. Therefore, Cardoso and Fonseca (2004) maximized the strain energy (F25), imposing kinematic functional requirements as constraints. However, enough strain energy in the elastic body is only a necessary condition to be a distributed CM but not a sufficient condition; therefore, the formulation cannot ensure the removal of point flexures in optimal topologies. In addition, the specifications of the design should be very carefully selected, e.g. kinematic constraints, input force and output forces need to be selected carefully to guarantee distributed CMs without point flexures. Lastly, a CM designed from this formulation has low ME since the stored strain energy is large inside the elastic body.

Wang (2009a) developed F26 to eliminate point flexures, arguing that the true optimum of the optimization problem as posed was a rigid-body linkage with revolute joints. More specifically, a kinematically permissible solution can generate large output displacement and has minimum strain energy; therefore, the optimization algorithm tends to generate solutions that closely imitate a rigid-body linkage by means of point flexures. Wang (2009a) also found three necessary conditions on the derived input stiffness matrix $\mathbf{K}_{\mathrm{I}}$, output stiffness matrix $\mathbf{K}_{\mathrm{O}}$ and strucrue stiffness matrix $\mathbf{K}_{\mathrm{S}}$ from the mechanism stiffness matrix $\mathbf{K}_{\mathrm{m}}$ which was ultimately from the global stiffness matrix. Both $\mathbf{K}_{\mathrm{I}}$ and $\mathbf{K}_{\mathrm{O}}$ have to be non-singular to ensure point flexure-free. For example, if any of $\mathbf{K}_{\mathrm{I}}$ and $\mathbf{K}_{\mathrm{O}}$ is singular, the $\mathbf{K}_{\mathrm{m}}$ is semi-definite so that the mechanism's structure permits an internal rigid-body displacement mode which results in point flexures in the optimal design. Thus, it is necessary to eliminate any rigid-body displacement mode in the $\mathrm{CM}$ to avoid any point flexures by maintaining $\mathbf{K}_{\mathrm{I}}$ and $\mathbf{K}_{\mathrm{O}}$ non-singular. These necessary conditions are formulated into F26. However, the presence of lumped compliance or point flexures cannot be completely prevented, since it similarly restrains the rigid-body motion. Wang (2009a) and Yin and Ananthasuresh (2003) shared the same idea, i.e. to restrain the motion; however, they did not change the tendency of lumped compliance. In addition, Deepak et al. (2009) also found that when the mechanical specification was not set properly, e.g. the desired GA was too large, point flexures still appeared in the optimum.

Lee (2011) designed CMs with a strain based formulation. $\mathrm{He}$ argued that in the conventional strain energy based formulation, an element with large strain but low material density had no priority to get more material distribution even though it was supposed to get more, which leaded to localized deformation or even point flexures. Thus, a strain based formulation (F21) was developed to eliminate this distortion by formulating the strain rather than strain energy of each element in the objective function. The new formulation reduced localized high deformation in CMs in some degree but the presence of point flexures was still hard to be avoided in the demonstrated examples.

The underlying reasons for the presence of point flexures in the topological design of CMs are still in debate in the literature, and a variety of formulations have been taken to deal with this problem. However, most of the formulations, though with more criteria, are still based on the conventional formulations, which tend to generate point flexures. Thus, no universal formulation in the literature completely avoids this problem. Efforts must be taken to find out new formulations which are not limited by the ideas behind conventional formulations.

\section{Conclusions and future work}

In the literature, CMs have been mostly designed for the balance of flexibility and stiffness, and maximized MA, GA or ME. However, design problems and formulations as defined with these attributes are not the main streams of design problems of mechanisms, i.e. FG, PG and MG. Thus, general Design Problems (DPA DPL) of CMs through the TO are defined in this paper due to the lack of comprehensive definitions for design problems of CMs in the literature. Typical design problems of rigid body mechanisms, i.e. FG, PG and $\mathrm{MG}$, are extended to the design problems of CMs based on DPA DPL.

Boundary conditions or working conditions that have been considered in the literature are also reviewed. The spring model captures the behaviour of actuators or work-piece and it has been widely accepted in the literature. However, the optimal results are highly dependent on the stiffness of springs (Wang, 2009b; Luo et al., 2005) so that the optimal CMs can only work properly with work-pieces which have the same stiffness as the one in the design specification, while in practice a CM may interact with different work-pieces. In addition, the modeling of work-pieces or actuators needs to be improved and rotational motions or forces need to be considered since only translational motions or forces have been considered in the literature.

Future work regarding boundary conditions lies in: (1) the accurate modeling of work-pieces in the case of path generation and motion generation CMs; (2) the design of CMs 
which are not sensitive to the stiffness of work-piece or actuators; (3) the design of CMs with variable boundaries conditions, e.g. consider input force as an design variable or support regions as an design variable, etc; (4) consider rotational inputs and outputs; (5) the design of CMs with specified actuators and output forces, i.e. the design specification (3).

Functional requirements and the associated formulations (F1-F25) in the literature are comprehensively reviewed along with their limitations. Based on whether the output is controlled quantitatively or not, the formulations can be categorized into two types: (1) formulations for quantitative design of CMs; (2) formulations for qualitative design of CMs. In addition, formulations that aim to solve the point flexure problem are also discussed. Formulations for the qualitative design of CMs cannot satisfy the defined design problems while formulations for the design of path following CMs partly satisfy the defined design problem in some degree although it is incomplete, and the improvement of the modeling of actuators and work-pieces is still in demand.

Lastly, an overview of the formulations for the point flexure problem is presented. Most of these formulations, although with more criteria, are still based on the conventional formulations, generating point flexures in results. Thus, no universal formulation yet in the literature ensures point flexure-free results. New formulations, point flexure-free by their nature, need to be developed in the future.

Table A1. List of abbreviations.

\begin{tabular}{ll}
\hline CM & Compliant Mechanism \\
TO & Topology Optimization \\
FCBPSS & Function, Context, Behaviour, \\
& Principle, Structure, State \\
ME & Mechanical Efficiency \\
MA & Mechanical Advantage \\
GA & Geometrical Advantage \\
FG & Function Generation \\
PG & Path Generation \\
MG & Motion Generation \\
DPA $(B, C, \ldots$. L) & Design Problem A $(B, C, \ldots$ L) \\
F1 $(2,3, \ldots, 25)$ & Formulation 1 $(2,3, \ldots, 25)$ \\
LSE & Least Square Error \\
MSE & Mutual Strain Energy \\
SE & Strain Energy \\
\hline
\end{tabular}

Acknowledgements. The first author wants to acknowledge the financial supports from the China Scholarship Council and the East China University of Science and Technology. The corresponding author wants to acknowledge the financial support received from the NSERC through a discovery grant. The first author also thanks Heather Mcwhinney for assisting in proof-reading this paper.

Edited by: Y. Li

Reviewed by: two anonymous referees

\section{References}

Ananthasuresh, G. K.: A new design paradigm for micro-electromechanical systems and investigations on the compliant mechanisms, Ph.D. thesis, University of Michigan, 1994.

Bendsoe, M. P. and Sigmund, O.: Topology Optimization: Theory, Methods and Applications, Springer, 2004.

Canfield, S. and Frecker, M.: Topology optimization of compliant mechanical amplifiers for piezoelectric actuators, Struct. Multidiscip. O., 20, 269-279, doi:10.1007/s001580050157, 2000.

Cardoso, E. L. and Fonseca, J. S. O.: Strain energy maximization approach to the design of fully compliant mechanisms using topology optimization, Lat. Am. J. Solids Stru., 1, 263-275, 2004.

Chen, S., and Wang, M. Y.: Designing distributed compliant mechanisms with characteristic stiffness, the ASME 2007 International Design Engineering Technical Conferences \& Computers and Information in Engineering Conference IDETC/CIE, 2007.

Deepak, S. R., Dinesh, M., Sahu, D. K., and Ananthasuresh, G. K.: A Comparative Study of the Formulations and Benchmark Problems for the Topology Optimization of Compliant Mechanisms, Journal of Mechanisms and Robotics, 1, 011003, doi:10.1115/1.2959094, 2009.

Du, H., Lau, G. K., Lim, M. K., and Qui, J.: Topological optimization of mechanical amplifiers for piezoelectric actuators under dynamic motion, Smar. Mat. St., 9, 788, doi:10.1088/09641726/9/6/308, 2000.

Frecker, M. and Canfield, S.: Optimal Design and Experimental Validation of Compliant Mechanical Amplifiers for Piezoceramic Stack Actuators, J. Intel. Mat. Syst. Str., 11, 360-369, doi:10.1106/d6uj-0fbt-p1w0-u1wx, 2000.

Frecker, M. I., Ananthasuresh, G. K., Nishiwaki, S., Kikuchi, N., and Kota, S.: Topological Synthesis of Compliant Mechanisms Using Multi-Criteria Optimization, J. Mech. Design, 119, 238, doi:10.1115/1.2826242, 1997.

Hetrick, J. A.: An energy efficiency approach for unified topological and dimensional synthesis of compliant mechanisms, Ph.D. thesis, University of Michigan, United States, Michigan, 158-158, 1999.

Howell, L. L.: Compliant Mechanisms, John Wiley \& Sons, New York, 2001.

Huang, S. C. and Lan, G. J.: Design and fabrication of a micro-compliant amplifier with a topology optimal compliant mechanism integrated with a piezoelectric microactuator, J. Micromech. Microeng., 16, 531-538, doi:10.1088/09601317/16/3/008, 2006.

Joo, J. and Kota, S.: Topological Synthesis of Compliant Mechanisms Using Nonlinear Beam Elements, Mech. Based Des. Struc., 32, 17-38, doi:10.1081/sme-120026588, 2004

Joo, J., Kota, S., and Kikuchi, N.: Topological Synthesis of Compliant Mechanisms Using Linear Beam Elements*, Mech. Struct. Mach., 28, 245-280, 2000.

Kikuchi, N., Nishiwaki, S., Fonseca, J. S. O., and Silva, E. C. N.: Design optimization method for compliant mechanisms and material microstructure, Comput. Method. Appl. M., 151, 401-417, 1998.

Kota, S., Joo, J., Li, Z., Rodgers, S., and Sniegowski, J.: Design of Compliant Mechanisms: Applications to MEMS, Analog Integr. Circ. S., 29, 7-15, doi:10.1023/a:1011265810471, 2001. 
Lau, G. K., Du, H., and Lim, M. K.: Use of functional specifications as objective functions in topological optimization of compliant mechanism, Comput. Method. Appl. M., 190, 4421-4433, doi:10.1016/S0045-7825(00)00325-X, 2001.

Lee, E.: A strain based topology optimization method, Ph.D. thesis, Rutgers The State University of New Jersey, New Brunswick, United States, New Jersey, 115 pp., 2011.

Lin, C. F. and Shih, C. J.: Topological optimum design of a compliant mechanism for planar optical modulator, Tamkang Journal of Science and Engineering, 5, 151-158, 2002.

Lin, Y. and Zhang, W. J.: Towards a novel interface design framework: function-behavior-state paradigm, Int. J. Hum.-Comput. St., 61, 259-297, doi:10.1016/j.ijhcs.2003.11.008, 2004.

Lu, K. J. and Kota, S.: Synthesis of shape morphing compliant mechanisms using a load path representation method, Smart Structures and Materials 2003, 5049, 337-348, 2003.

Luo, Z. and Zhang, N.: A multi-criteria topology optimization for systematic design of compliant mechanisms, CMC-Comput. Mater. Con., 28, 27-55, 2012.

Luo, Z., Chen, L., Yang, J., Zhang, Y., and Abdel-Malek, K.: Compliant mechanism design using multi-objective topology optimization scheme of continuum structures, Struct. Multidiscip. O., 30, 142-154, doi:10.1007/s00158-004-0512-y, 2005.

Min, S. and Kim, Y.: Topology Optimization of Compliant Mechanism with Geometrical Advantage, JSME Int. J. C-Mech. Sy., 47, 610-615, 2004.

Nishiwaki, S., Min, S., Yoo, J., and Kikuchi, N.: Optimal structural design considering flexibility, Comput. Method. Appl. M., 190, 4457-4504, 2001.

Norton, R. L.: Design of Machinery: An Introduction to the Synthesis and Analysis of Mechanisms and Machines, McGraw-Hill College, 2003.

Pedersen, C. B. W., Buhl, T., and Sigmund, O.: Topology synthesis of large-displacement compliant mechanisms, Int. J. Numer. Meth. Eng., 50, 2683-2705, doi:10.1002/nme.148, 2001.

Rahmatalla, S. and Swan, C. C.: Sparse monolithic compliant mechanisms using continuum structural topology optimization, Int. J. Numer. Meth. Eng., 62, 1579-1605, doi:10.1002/nme.1224, 2005.

Rai, A. K., Saxena, A., and Mankame, N. D.: Synthesis of Path Generating Compliant Mechanisms Using Initially Curved Frame Elements, J. Mech. Design, 129, 1056, doi:10.1115/1.2757191, 2007.

Rai, A. K., Saxena, A., and Mankame, N. D.: Unified synthesis of compact planar path-generating linkages with rigid and deformable members, Struct. Multidiscip. O., 41, 863-879, doi:10.1007/s00158-009-0458-1, 2009.
Saggere, L. and Kota, S.: Synthesis of Planar, Compliant FourBar Mechanisms for Compliant-Segment Motion Generation, J. Mech. Design, 123, 535-541, 2001.

Saxena, A.: Synthesis of Compliant Mechanisms for Path Generation using Genetic Algorithm, J. Mech. Design, 127, 745, doi:10.1115/1.1899178, 2005.

Saxena, A. and Ananthasuresh, G. K.: An optimality criteria approach for the topology synthesis of compliant mechanisms, ASME Deign Engineering Technical Conferences, Atlanta, Georgia, USA, 1998.

Saxena, A. and Ananthasuresh, G. K.: On an optimal property of compliant topologies, Struct. Multidiscip. O., 19, 36-49, doi:10.1007/s001580050084, 2000.

Saxena, A. and Ananthasuresh, G. K.: Topology Synthesis of Compliant Mechanisms for Nonlinear Force-Deflection and Curved Path Specifications, J. Mech. Design, 123, 33, doi:10.1115/1.1333096, 2001.

Sigmund, O.: On the Design of Compliant Mechanisms Using Topology Optimization*, Mech. Struct. Mach., 25, 493-524, doi:10.1080/08905459708945415, 1997.

Sigmund, O.: Optimum Design of Microelectromechanical Systems, in: Mechanics for a New Mellennium, edited by: Aref, H. and Phillips, J., Springer Netherlands, 505-520, 2002.

Tai, K., Cui, G. Y., and Ray, T.: Design Synthesis of Path Generating Compliant Mechanisms by Evolutionary Optimization of Topology and Shape, J. Mech. Design, 124, 492, doi:10.1115/1.1480818, 2002.

Ullah, I. and Kota, S.: Optimal synthesis of mechanisms for path generation using Fourier descriptors and global search methods, J. Mech. Design, 119, 504-510, 1997.

Wang, M. Y.: A Kinetoelastic Formulation of Compliant Mechanism Optimization, Journal of Mechanisms and Robotics, 1, 021011-021010, 2009a.

Wang, M. Y.: An analysis of the compliant mechanism models, Reconfigurable Mechanisms and Robots, 2009, ReMAR 2009 ASME/IFToMM International Conference on, 2009b, 377-385, 2009b.

Wang, M. Y.: Mechanical and geometric advantages in compliant mechanism optimization, Frontiers of Mechanical Engineering in China, 4, 229-241, doi:10.1007/s11465-009-0066-1, 2009c.

Yin, L. and Ananthasuresh, G. K.: Design of Distributed Compliant Mechanisms, Mech. Based Des. Struc., 31, 151-179, doi:10.1081/sme-120020289, 2003.

Zhou, H. and Ting, K. L.: Topological synthesis of compliant mechanisms using spanning tree theory, J. Mech. Design, 127, 753759,2005 